\title{
Three-dimensional photoacoustic tomography of small animal brain with a curved array transducer
}

Xinmai Yang, Lihong V. Wang, Anastasios Maurudis, John Gamelin, Andres Aguirre, et al.

Xinmai Yang, Lihong V. Wang, Anastasios Maurudis, John Gamelin, Andres Aguirre, Quing Zhu, "Three-dimensional photoacoustic tomography of small animal brain with a curved array transducer," Proc. SPIE 7177, Photons Plus Ultrasound: Imaging and Sensing 2009, 71770K (12 February 2009); doi: $10.1117 / 12.809590$

SPIE. Event: SPIE BiOS, 2009, San Jose, California, United States 


\title{
Three-dimensional photoacoustic tomography of small animal brain with a curved array transducer
}

\author{
Xinmai Yang* and Lihong V. Wang \\ Optical Imaging Laboratory, Department of Biomedical Engineering, Washington University in St. \\ Louis, St. Louis, Missouri, 63130 \\ Anastasios Maurudis, John Gamelin, Andres Aguirre and Quing Zhu \\ Dept. of Electrical Engineering, University of Connecticut, Storrs, CT 06269
}

\begin{abstract}
We present the application of an optimized curved array photoacoustic tomographic imaging system, which can provide rapid, high-resolution photoacoustic imaging of small animal brains. The system can produce a B-mode, 90-degree field-of-view image at sub-200 $\mu \mathrm{m}$ resolution at a frame rate of $\sim 1$ frame/second when a $10-\mathrm{Hz}$ pulse repetition rate laser is employed. By rotating samples, a complete 360-degree scan can be achieved within 15 seconds. In previous work, two-dimensional ex vivo mouse brain cortex imaging has been reported. In the current work, we report threedimensional small animal brain imaging obtained with the curved array system. The results are presented as a series of two-dimensional cross-sectional images. Besides structural imaging, the blood oxygen saturation of the animal brain cortex is also measured in vivo. In addition, the system can measure the time-resolved relative changes in blood oxygen saturation level in the small animal brain cortex. Finally, ultrasonic gel coupling, instead of the previously adopted water coupling, is conveniently used in near-real-time $2 \mathrm{D}$ imaging.
\end{abstract}

Keywords: photoacoustic tomography, image reconstruction, array, brain

\section{INTRODUCTION}

In recent years, photoacoustic imaging (PAI) has emerged as a promising novel biomedical imaging modality ${ }^{1-5}$. As a hybrid imaging modality, PAI can provide ultrasound-resolution images with intrinsic optical contrast in regions up to 5 $\mathrm{cm}$ deep $^{6,7}$. Besides structural information, PAI can also detect functional changes and disorders in vivo ${ }^{8,9}$ since these changes and disorders usually induce local optical contrast through changing blood volume and oxygenation. Therefore, PAI represents a novel technology from the perspectives of both ultrasound and optical imaging: it adds new contrast and functional information to ultrasound imaging, and greatly extends the depth of high resolution optical imaging.

In the past decade, as small animal models have been established for many human diseases, in vivo small animal imaging techniques have developed dramatically. PAI has attracted much attention in this area due to the aforementioned merits. In particular, reconstruction-based PAI-photoacoustic tomography (PAT), has been successfully applied to small animal brain imaging, and its applications in structural, functional, and molecular imaging have been demonstrated ${ }^{8-10}$.

Frequently, PAT involves the scanning of a single ultrasound detector. The use of a single detector is inexpensive and can provide a good signal-to-noise ratio (SNR) for an image; however, the data acquisition duration is long, usually $\sim 20$ minutes per cross-section. The prolonged measurement time presents great challenges for the control of small animal physiological parameters, especially when time-resolved functional information is desired or multiple slices of cross-

\footnotetext{
${ }^{*}$ Current Address: KU Bioengineering Research Center, Department of Mechanical Engineering, the University of Kansas, Lawrence, KS 66045
}

Photons Plus Ultrasound: Imaging and Sensing 2009, edited by Alexander A. Oraevsky, Lihong V. Wang, Proc. of SPIE Vol. 7177, 71770K · C 2009 SPIE · CCC code: 1605-7422/09/\$18 · doi: 10.1117/12.809590 
section images are needed. Therefore, real-time or near real-time PAT scans are necessary, which may be realized by using array transducers.

The use of ultrasound array transducers for PAT has been explored by several groups ${ }^{11-18}$. In these studies, linear or curved array transducers were employed to reduce data acquisition time and meet clinical needs. However, these systems are neither designed nor optimized for small animal use. We have developed a curved array photoacoustic system optimized for high-resolution tomography of small animal brains ${ }^{19}$. The array was custom fabricated by Imasonic, Inc. (Besançon, France) using piezocomposite technology for high sensitivity and SNR. The system employs 128-element ultrasonic transducers operating at $5-\mathrm{MHz}$ with $80 \%$ bandwidth for resolution of fine features such as brain vasculatures, while retaining high sensitivity for deep imaging. To mimic the scanning of a single element transducer, all the elements are arranged to form a quarter circle of $25 \mathrm{~mm}$ radius, therefore, it takes 3 rotations to get the 360degree view data. Each individual element has an elevation height of $10 \mathrm{~mm}$ with an azimuthal pitch of one wavelength $(0.308 \mathrm{~mm})$ and a kerf of $0.1 \mathrm{~mm}$. Furthermore, each element is cylindrically focused with a focal distance of $19 \mathrm{~mm}$. In the case of 360-degree scan, this focusing effect results in a uniform central imaging region of approximate $16 \mathrm{~mm}$ in diameter. A 16-channel data-acquisition module and dedicated channel detection electronics allow capture of a 90degree field-of-view image in less than one second when a $10-\mathrm{Hz}$ pulse repetition rate laser is employed, and a complete 360 -degree scan can be achieved through sample rotation within 15 seconds.

The systematic evaluation of this system has been reported in detail previously ${ }^{19,20}$. In this article, we report the applications of the system on small animal brain imaging, including 3D cross-sectional brain imaging, in vivo brain cortex imaging, and hemodynamic measurements on a rat brain cortex. The results suggest that the current system can perform PAT of small animal brain to provide both structural and functional information in vivo.

\section{METHODS AND MATERIALS}

The schematic for the noninvasive photoacoustic tomography of rat brains is shown in Figure 1. A Q-switched Nd:YAG laser (LS-2137/2, LOTIS TII)-pumped tunable Ti:sapphire laser (LT-2211A, LOTIS TII) was employed to provide laser pulses with a FWHM $<15 \mathrm{~ns}$, a pulse repetition rate of $10 \mathrm{~Hz}$, and a wavelength range of 750 to $820 \mathrm{~nm}$. The incident energy density of the laser beam was controlled to be less than $15 \mathrm{~mJ} / \mathrm{cm}^{2}$ on the surface of the animal head, which is well below the ANSI limit ${ }^{21}$. The beam was diverged with a concave lens and homogenized by a circular diffuser to produce a uniform illumination of approximately $20 \mathrm{~mm}$ in diameter at the sample. The laser light was positioned at the center of curvature of the transducer and illuminated the sample orthogonal to the imaging plane of the transducer for maximum uniformity.

In the animal experiments, Sprague Dawley rats (60-100 gram body weight) or Swiss Webster mice ( 25 gram body weight) (From Harlan Sprague Dawley, Incorporated, Indianapolis, Indiana) were used. For in vivo tests, a small animal was initially anesthetized by the intramuscular injection of a mixture of $87 \mathrm{mg} / \mathrm{kg}$ ketamine plus $13 \mathrm{mg} / \mathrm{kg}$ xylazine. Subsequent anesthesia was achieved by the inhalation of a mixture of $\mathrm{O}_{2}$ and isoflurane. Before experiments, the hair on the head of the small animal was depilated using hair removal lotion. The mouth and nose of the animal were covered with a breathing mask to allow it to breathe in water and to deliver anesthesia gas. During experiments, the small animal was mounted on a rotary stage positioned at the center of the curved array transducer. The rotary stage could be turned in $90^{\circ}$ increments to simulate the angular view of a full ring array. The head of the small animal was adjusted so that the brain cortex surface was parallel with the imaging plane. After the data acquisition for PAT, the animal was sacrificed by the intraperitoneal injection of highly concentrated pentobarbital.

In the measurements of blood oxygenation, we assumed that deoxyhemoglobin $(\mathrm{Hb})$ and oxyhemoglobin $\left(\mathrm{HbO}_{2}\right)$ were the dominant absorbing compounds in blood at two wavelengths $\lambda_{1}$ and $\lambda_{2}$. Relative total hemoglobin concentration $(\mathrm{rHbT})$ and blood oxygen saturation $\left(\mathrm{SO}_{2}\right)$ could then be calculated using the detected optical absorptions at the two applied wavelengths ${ }^{22-24}$ :

$$
\mathrm{rHbT}=\left[\mathrm{HbO}_{2}\right]+[\mathrm{Hb}]=\frac{\mu_{a}^{\lambda_{1}} \Delta \varepsilon_{H b}^{\lambda_{2}}-\mu_{a}^{\lambda_{2}} \Delta \varepsilon_{H b}^{\lambda_{1}}}{\varepsilon_{H b}^{\lambda_{1}} \varepsilon_{H b O_{2}}^{\lambda_{2}}-\varepsilon_{H b}^{\lambda_{2}} \varepsilon_{H b O_{2}}^{\lambda_{1}}},
$$




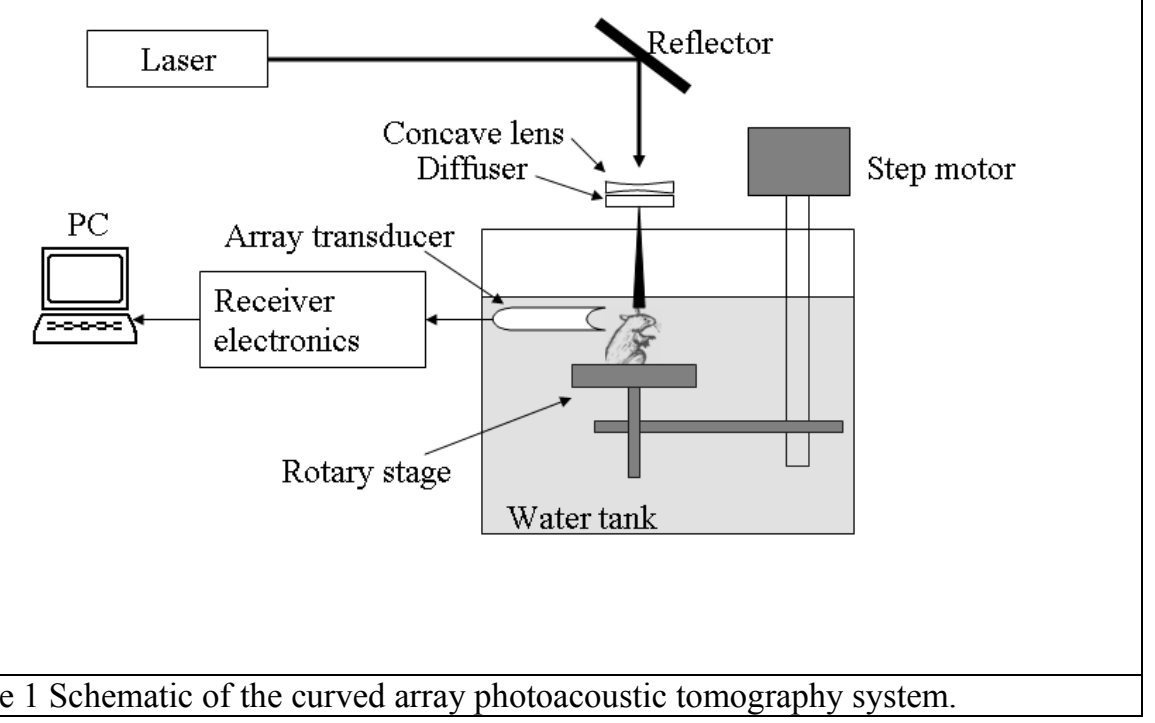

$\mathrm{SO}_{2}=\frac{\left[\mathrm{HbO}_{2}\right]}{\left[\mathrm{HbO}_{2}\right]+[\mathrm{Hb}]}=\frac{\mu_{a}^{\lambda_{2}} \varepsilon_{H b}^{\lambda_{1}}-\mu_{a}^{\lambda_{1}} \varepsilon_{H b}^{\lambda_{2}}}{\mu_{a}^{\lambda_{1}} \Delta \varepsilon_{H b}^{\lambda_{2}}-\mu_{a}^{\lambda_{2}} \Delta \varepsilon_{H b}^{\lambda_{1}}}$,

where $\mu_{a}$ is the absorption coefficient; $\varepsilon_{\mathrm{Hb}}$ and $\varepsilon_{\mathrm{HbO}_{2}}$ are the known molar extinction coefficients of $\mathrm{Hb}$ and $\mathrm{HbO}_{2}$, respectively; $\Delta \varepsilon_{\mathrm{Hb}}=\varepsilon_{\mathrm{HbO}_{2}}-\varepsilon_{\mathrm{Hb}}$; and $[\mathrm{Hb}]$ and $\left[\mathrm{HbO}_{2}\right]$ are the concentrations of the two forms of hemoglobin, respectively. By irradiating an animal head with lights at two different wavelengths, $\lambda_{1}$ and $\lambda_{2}$, independently, we could get two photoacoustic images that represent the distributions of the optical energy deposition in the cerebral cortex corresponding to the two wavelengths. The optical energy deposition is dependent on the optical absorption and the light fluence at specific location. Considering that the skin and skull covering the brain are relatively homogeneous, the light fluence in the brain cerebral cortex is similarly homogeneously distributed in the horizontal plane at the two wavelengths. We can then calculate the images of an absolute estimation of $\mathrm{SO}_{2}$ based on Eqn (2).

\section{RESULTS}

Three-dimensional PAT brain images of a mouse were obtained non-invasively as a series of 2-dimensional crosssections. The laser wavelength used was $797 \mathrm{~nm}$. Figure 2 shows the PAT cross-sections at different brain horizontal planes. Figure 2a shows the cortex surface. Figures $2 \mathrm{~b}$-e show the interior brain structures underneath the superficial cortex with a $2 \mathrm{~mm}$ increment along the depth. Because of the breathing motion effects, we show only the in situ experimental results. To illustrate the features shown on these PAT images, we have labeled some characteristic tissue structures. An MRI image at a similar depth as Figure $2 \mathrm{~d}$ is presented in Figure $2 \mathrm{f}$ and shows good similarities. In Figure $2 \mathrm{~d}$, the ventricular system is clearly visible on the image. 
a)

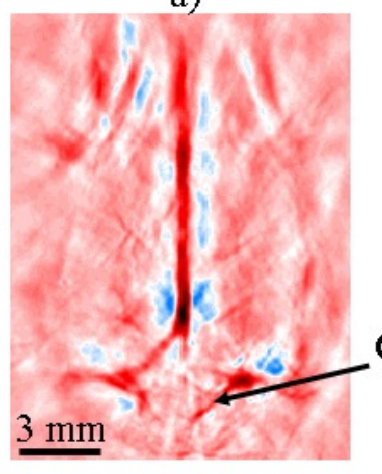

d)

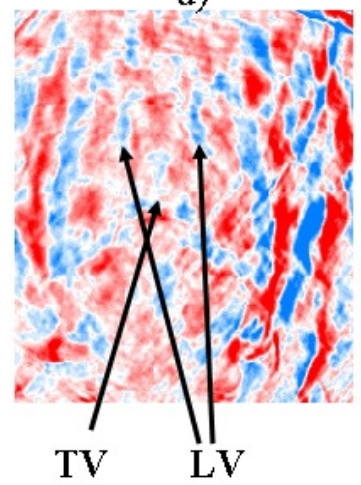

b)

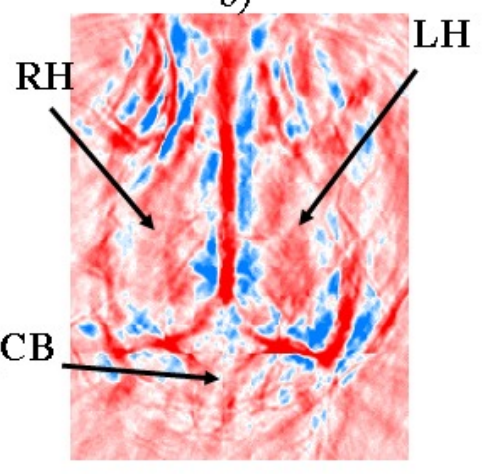

e)

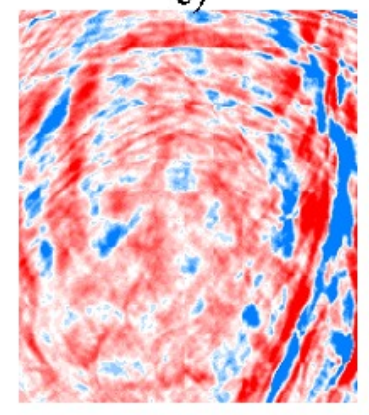

Optical absorption c)

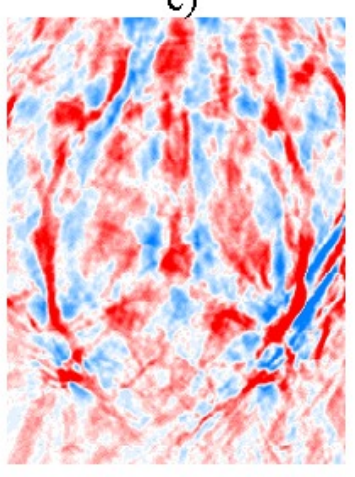

f)

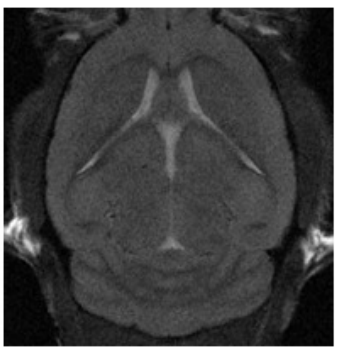

Min

Max

Figure 2 In situ 3D mouse brain images obtained by the curved array photoacoustic tomography system. The images show horizontal cross-sections from the dorsal to the ventral part of the brain, where the imaging depth is a), $0 \mathrm{~mm}$; ), $2.0 \mathrm{~mm}$; c), $4 \mathrm{~mm}$; d), $6 \mathrm{~mm}$; and e), $8.0 \mathrm{~mm}$ from the top surface of the mouse's brain, with a interval of $2 \mathrm{~mm}$. Major tissue structures are indicated on the images. An MRI image at a similar depth as d) is shown in $\mathrm{f}$ ). The color bar shows the relative magnitude of optical absorption. CB: Cerebellum; RH: Right hemisphere; LH: Left hemisphere; LV: Lateral ventricle; TV: Third ventricle.

To further demonstrate the capability of the current system to potentially identify the cerebral ventricular system, we imaged the brain of a hydrocephalic mouse, with the result in Figure 3. From the images, there are no identifiable structures inside the brain of a hydrocephalic mouse, which is consistent with the fact that these animals have abnormal enlarged brain ventricles.

In the above 3D cross-sectional brain image, since the mouth and nose of the small animals block the ultrasound signals from the tissue structures underneath the brain cortex surface, we rotated the animal head only twice for a 270 degree view angle.

By rotating the sample, better quality images can be obtained, however, the data acquisition speed is reduced. To get near real-time imaging, we avoided rotation. With the current quarter-ring array, we can have a 90-degree view angle if no rotation is employed. Although the image quality is degraded and some information may be missing because of the incomplete data captured with no rotation, there are distinct advantages. First, near real-time PAT images can be acquired, which allow us to monitor the time-resolved change in a small animal. Second, instead of using water as the coupling material between the transducer array and a sample, ultrasound gel can be used as the coupling medium 
without worrying about coupling problems caused by rotation. In PAT experiments reported in literatures, water tanks have been used to hold water for coupling while the detection transducer was scanned. The use of ultrasound gel is much more convenient. Furthermore, although with 90-degree view angle, the information on the image is incomplete, we can focus on the structures of interest one at a time without monitoring the whole image.
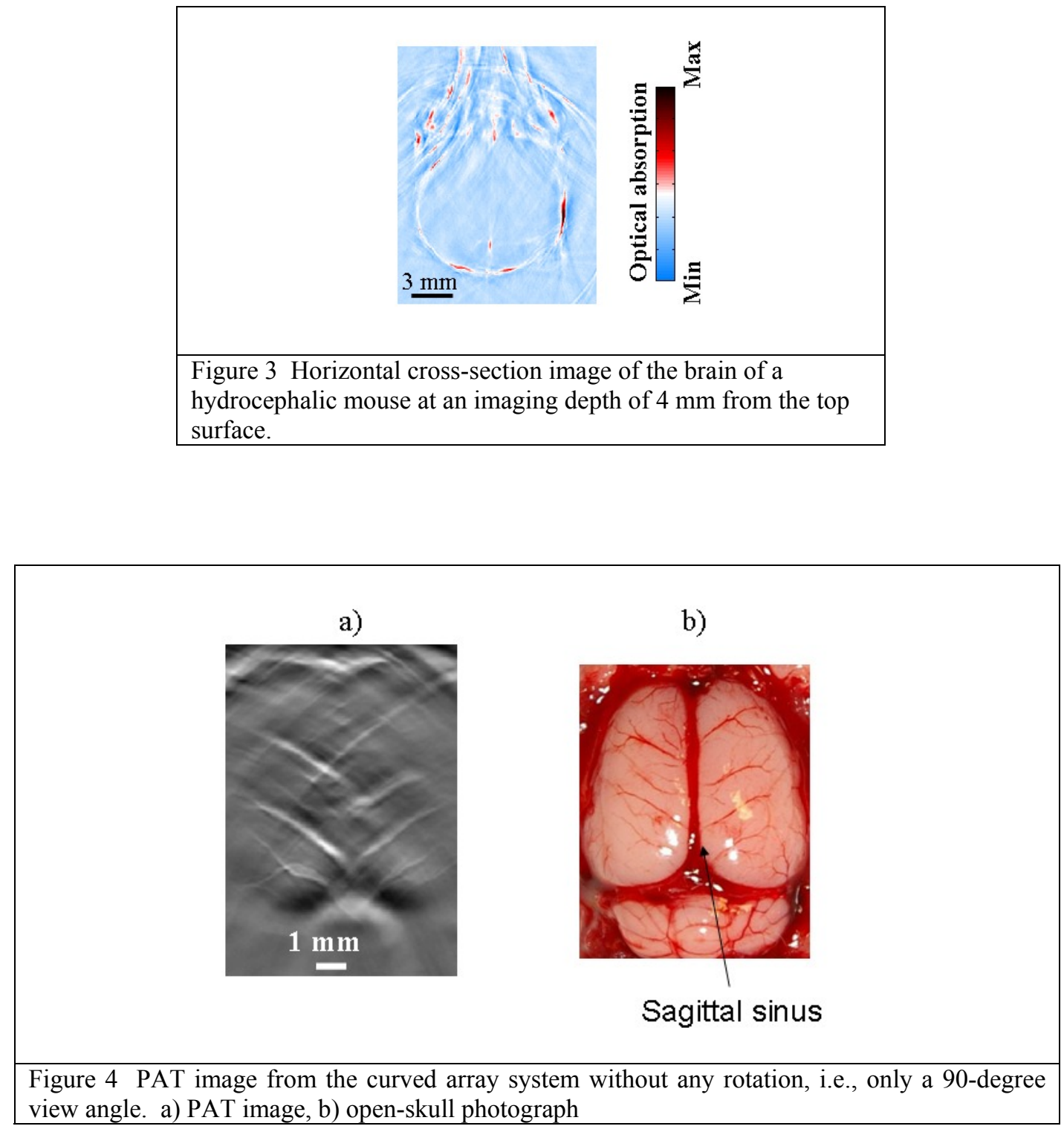

Figure 4 shows a rat brain cortex image obtained with the curved array without rotating the transducer. Because only 90 degree view angle data was acquired, some structures are missing from the image, including, notably, the sagittal sinus, which is labeled on the open skull photograph. However, Figure 4a still shows most of the blood vessels on the cortical surface, which are oriented perpendicular or nearly perpendicular to the sagittal sinus, and exhibits a good match with the open skull photograph shown in Figure 4b. Most important, this PAT image was acquired within $\sim 1 \mathrm{~s}$, which is nearly real-time monitoring. Therefore, with the current curved array, we can provide fast-frame images at $\sim 1$ frame/second. The fast frame rate allows us to monitor the changes in these blood vessels in Figure $4 \mathrm{a}$ as a function of time. 
Figure 5 shows the measured photoacoustic signal strength as a function of time as the blood oxygenation level on a rat brain cortex changes. The signal strength was obtained through integration of signals over each image. The animal experienced two physiological statuses: normoxia and hyperoxia. Figure 5a shows the hemodynamic changes on the rat brain cortex when the physiological status changed from normoxia to hyperoxia, and Figure $5 \mathrm{~b}$ shows the hemodynamic result when it changed from hyperoxia to normoxia. The laser wavelength used was $771 \mathrm{~nm}$. At this wavelength, deoxyhemoglobin has a greater extinction than oxyhemoglobin. Because the hyperoxic state carries less deoxyhemoglobin than the normoxic state, we observed a decrease on the strength of the photoacoustic signals in Figure $5 \mathrm{a}$ and an increase on the strength of the photoacoustic signals in Figure $5 \mathrm{~b}$.
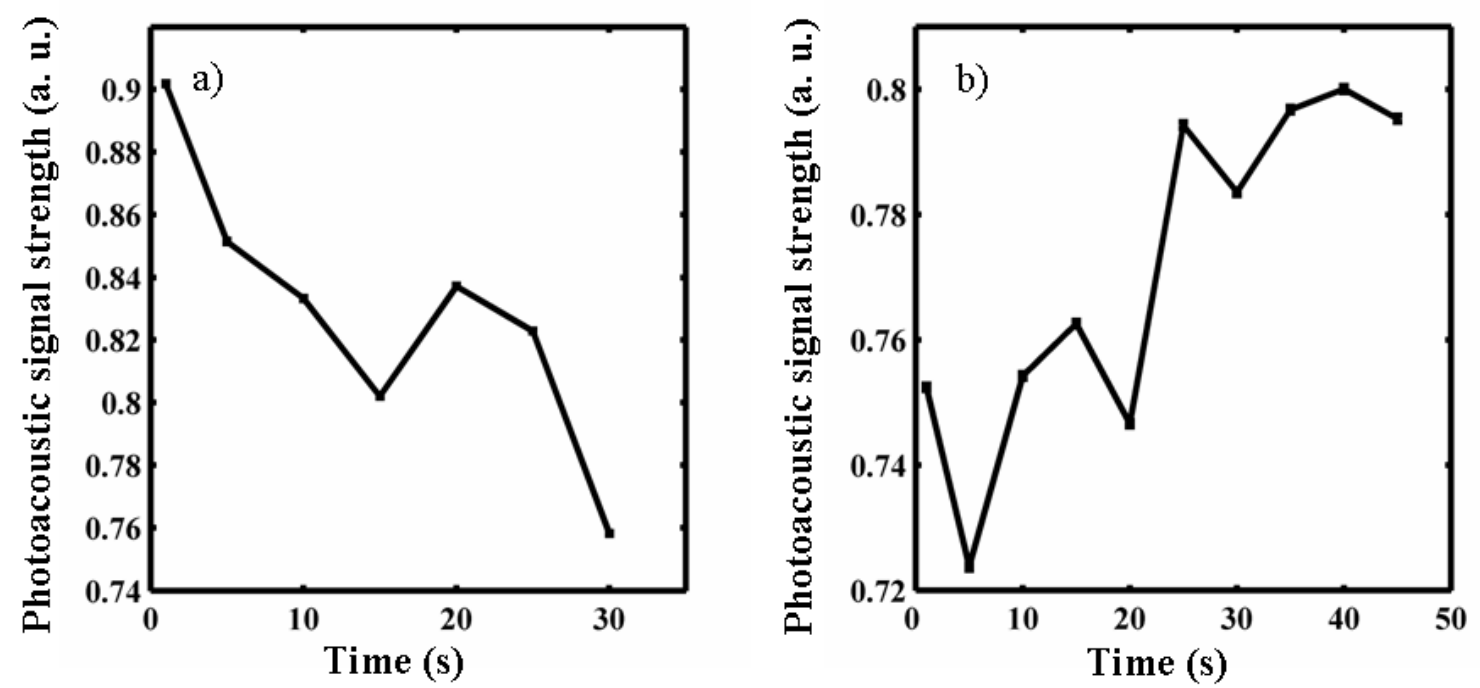

Figure 5 Rat brain cortex hemodynamics with the curved array transducer. a) the photoacoustic signal strength change on the rat brain cortex when the physiological status changed from normoxia to hyperoxia; $b$ ) the photoacoustic signal strength change when the physiological status changed from hyperoxia to normoxia. The laser wavelength was $771 \mathrm{~nm}$. The error bars are the standard errors at each measurement point. 

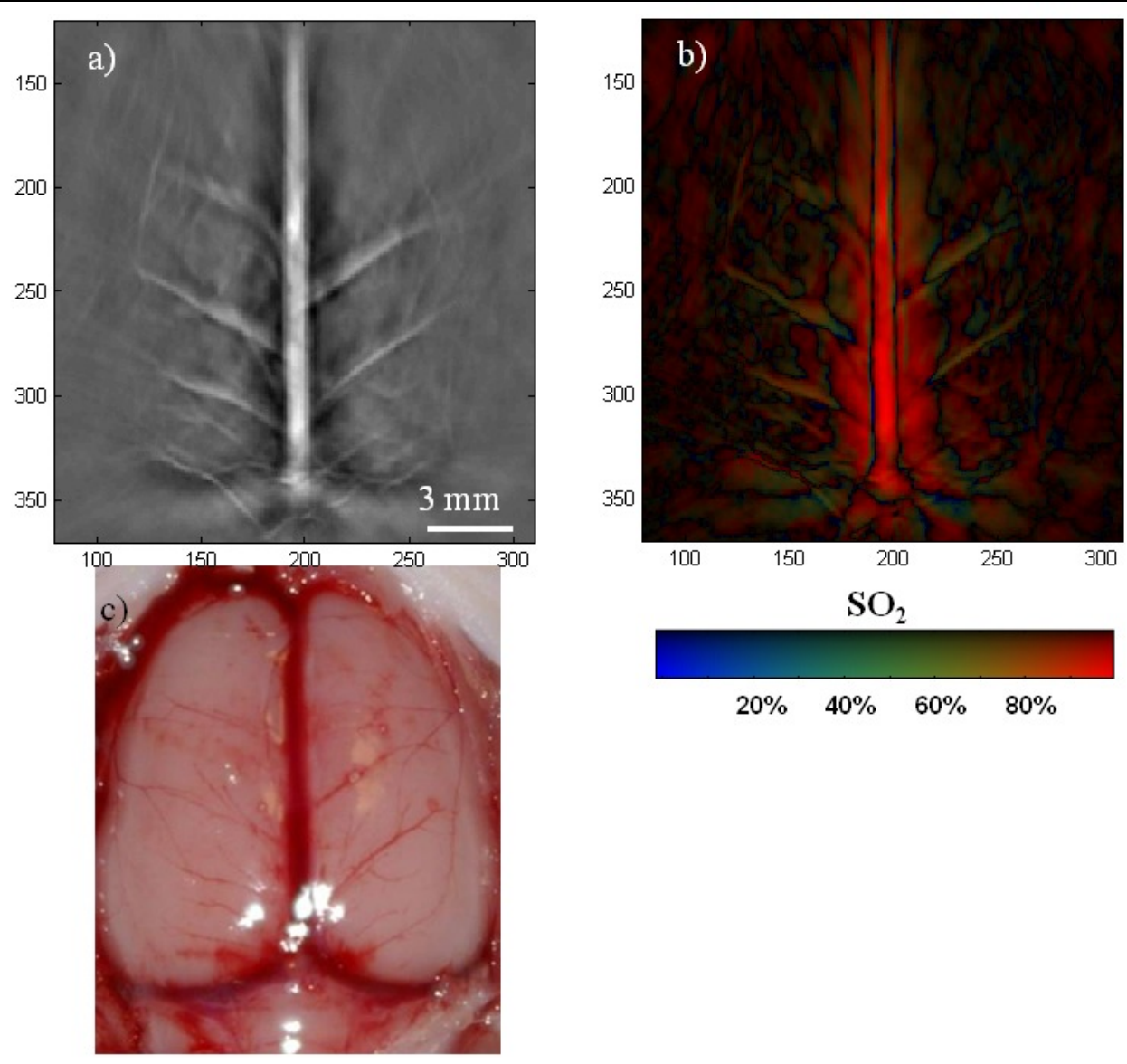

Figure 6 The in vivo measurement of a rat brain cortex with a $270^{\circ}$ scan angle. a) structural image; b) $\mathrm{SO}_{2}$ distribution on the rat brain cortex; c) open-skull photograph. The $\mathrm{SO}_{2}$ values were calculated from the blood vessels shown on cortex surface

Figure 6 shows an in vivo measurement of a rat ( 90 gram body weight) brain cortex with a $270^{\circ}$ scan angle, i.e., the animal was rotated twice to receive photoacoustic signals generated at the brain cortex from a $270^{\circ}$ angle. Figure $6 \mathrm{a}$ shows the structural image, which matches very well with the open skull photograph shown in Figure 6c. To obtain the $\mathrm{SO}_{2}$ distribution on the rat brain cortex, we acquired images with laser wavelengths of $797 \mathrm{~nm}$ and $762 \mathrm{~nm}$. At the isosbestic $797 \mathrm{~nm}$ wavelength, deoxy- and oxyhemoglobin have the same molar optical extinction coefficients. At 762 $\mathrm{nm}$, the optical extinction coefficient of deoxyhemoglobin is about 2.5 times of that of oxyhemoglobin. At each scanning position, images were acquired in one minute for both wavelengths, and then the $\mathrm{SO}_{2}$ values were calculated by Eqn. (2). Figure $6 \mathrm{~b}$ shows calculated $\mathrm{SO}_{2}$ on the rat brain cortex. The results show reasonable $\mathrm{SO}_{2}$ distributions on the rat brain cortex with most large blood vessels on the brain cortex having low $\mathrm{SO}_{2}$ values. This measurement is consistent with the fact that the vessels shown on the image close to the sagittal sinus are draining veins.

\section{DISCUSSION}

We have applied an optimized curved array photoacoustic system developed previously to small animal brain imaging. The system has been demonstrated with resolutions below $200 \mu \mathrm{m}^{19,20}$. In this paper, we reported that nearly real-time imaging with this system is possible. Additionally, ultrasonic gel coupling, instead of water coupling, was used in obtaining the nearly real-time images. Using the new system, we have demonstrated in vivo small animal brain cortex imaging. The blood oxygen saturation level on a small animal brain cortex has also been measured. The time-resolved 
curve in the relative change of blood oxygen saturation level was presented when the animal physiological status changes between hyperoxia and normoxia. An in situ, three-dimensional, whole brain image with the new system was also shown and compared with MRI images. The results demonstrated that the curved array system is capable of producing reliable PA images for small animal brain study

\section{ACKNOWLEDGEMENTS}

We thank Song $\mathrm{Hu}$ for helpful discussion on $\mathrm{SO}_{2}$ calculation. This project was sponsored in part by National Institutes of Health Grant Nos. R01 NS46214 (BRP) and R01 EB000712

\section{REFERENCE}

${ }^{1}$ C. G. A. Hoelen, F. F. M. de Mul, R. Pongers, and A. Dekker, "Three dimensional photoacoustic imaging of blood vessels in tissue," Opt. Lett. 23, 648-650 (1998).

${ }^{2}$ R. A. Kruger, D. R. Reinecke, and G. A. Kruger, "Thermoacoustic computed tomographytechnical considerations," Med. Phys. 26, 1832-1837 (1999).

${ }^{3}$ R. O. Esenaliev, A. A. Karabutov, and A. A. Oraevsky, "Sensitivity of laser opto-acoustic imaging in detection of small deeply embedded tumors," IEEE J. Sel. Top. Quantum Electron. 5, 981-988 (1999).

${ }^{4}$ K. P. Köstli, D. Frauchiger, J. J. Niederhauser, G. Paltauf, H. P. Weber, and M. Frenz, "Optoacoustic imaging using a three-dimensional reconstruction algorithm," IEEE J. Sel. Top. Quantum Electron. 7, 918-923 (2001).

${ }^{5} \mathrm{M}$. Xu and L.-H. Wang, "Biomedical photoacoustics," Review of Scientific Instruments, 77 (4), 041101 (2006).

${ }^{6} \mathrm{G}$. $\mathrm{Ku}$ and $\mathrm{L}$. V. Wang, "Deeply penetrating photoacoustic tomography in biological tissues enhanced with an optical contrast agent," Opt. Lett. 30, 507-509 (2005).

${ }^{7}$ G. Ku, B. D. Fornage, X. Jin, M. Xu, K. K. Hunt, and L. V. Wang, "Thermoacoustic and photoacoustic tomography of thick biological tissues toward breast imaging," Technology in Cancer Research \& Treatment 4, 559-566 (2005).

${ }^{8}$ X. Wang, Y. Pang, G. Ku, X. Xie, G. Stoica, and L. V. Wang, "Noninvasive laser-induced photoacoustic tomography for structural and functional imaging of the brain in vivo," Nat. Biotechnol., 21, 803-806 (2003).

${ }^{9}$ M. Li, J. Oh, X. Xie, G. Ku, W. Wang, C. Li, G. Lungu, G. Stoica, and L. V. Wang, "Simultaneous molecular and hypoxia imaging of brain tumors in vivo using spectroscopic photoacoustic tomography," Proceedings of the IEEE, 96 481-489 (2008).

${ }^{10}$ H. F. Zhang, K. Maslov, G. Stoica, and L.-H. Wang, "Functional photoacoustic microscopy for high-resolution and noninvasive in vivo imaging," Nat. Biotechnol. 24, 848-851 (2006).

${ }^{11}$ V. Kozhushko, T. Khokhlova, A. Zharinov, I. Pelivanov, V. Solomatin, \& A. Karabutov, "Focused array transducer for two-dimensional optoacoustic tomography," J Acoust Soc Am 116, 1498-1506 (2004).

${ }^{12}$ R. A. Kruger, W. L. Kiser, D. R. Reinecke, \& G. A. Kruger, "Thermoacoustic computed tomography using a conventional linear transducer array," Med Phys 30, 856-860 (2003).

${ }^{13}$ S. Park, S. Mallidi, A. Karpiouk, S. Alyamov, \& S. Emelianov, "Photoacoustic imaging using array transducer," Proc. SPIE 6437, 643714 (2007). 
${ }^{14}$ B. Yin, D. Xing, Y. Wang, Y. Zeng, Y. Tan, \& Q. Chen, "Fast photoacoustic imaging system based on 320-element linear transducer array," Phys Med Biol 49, 1339-1346 (2004).

${ }^{15}$ R. J. Zemp, R. Bitton, M. L. Li, K. K. Shung, G. Stoica, \& L. V. Wang, "Photoacoustic imaging of the microvasculature with a high-frequency ultrasound array transducer," $J$ Biomed Opt 12, 010501 (2007).

${ }^{16}$ J. J. Niederhauser, M. Jaeger, R. Lemor, P. Weber, \& M. Frenz, "Combined ultrasound and optoacoustic system for real-time high-contrast vascular imaging in vivo," IEEE Trans Med Imaging 24, 436-440 (2005).

${ }^{17}$ A. A. Oraevsky \& A. Karabutov, "Ultimate sensitivity of time-resolved opto-acoustic detection," Proc. SPIE 3916, 228-239 (2000).

${ }^{18}$ R. A. Kruger, W. L. Kiser, D. R. Reinecke, G. A. Kruer, \& K. D. Miller, "Thermoacoustic molecular imaging of small animals," Molecular Imaging 2, 113-122 (2003).

${ }^{19}$ J. Gamelin, A. Aguirre, A. Maurudis, F. Huang, D. Castillo, L. V. Wang, and Q. Zhu, "Curved array photoacoustic tomographic system for small animal imaging,” J Biomed Opt. Mar-Apr;13, 024007 (2008).

${ }^{20}$ F. Huang, A. Maurudis, J. Gamelin,, A. Aguirre, D. Castillo, P. Guo, and Q. Zhu, "A fast photoacoustic imaging system based on a curved ultrasound transducer array," 2007 IEEE 33rd Annual Northeast Bioengineering Conference Pages: 47-8 (2007).

${ }^{21}$ Laser Institute of America, American National Standard for Safe Use of Lasers ANSI Z136.12000, American National Standards Institute Inc., New York, NY, 2000.

${ }^{22}$ B. Chance, E. Borer, A. Evans, G. Holtom, J. Kent, M. Maris, K. Mccully, J. Northrop, and M. Shinkwin, "Optical and nuclearmagnetic- resonance studies of hypoxia in human tissue and tumors," Ann. N.Y. Acad. Sci. 551, 1-16 (1988).

${ }^{23}$ R. L. Barbour, A. Gebrewold, and B. M. Altura, "Optical spectroscopy and cerebral vascular effects of alcohol in the intact brain: effects on tissue deoxyhemoglobin, blood content, and reduced cytochrome oxidase," Alcohol Clin. Exp. Res. 17, 1319-1324 (1993).

${ }^{24}$ H. Liu, D. A. Boas, Y. Zhang, A. G. Yodh, and B. Chance, "Determination of optical properties and blood oxygenation in tissue using continuous NIR light," Phys. Med. Biol. 40, 1983-1993 (1995). 